Literatura y Lingüística $\mathrm{N}^{\circ} 25$

ISSN 0716-5811 / pp. 249-273

\title{
Estudio lingüístico-comparativo del siciliano y el español*
}

\author{
Eva Núñez Méndez** \\ Raven Chakerian***
}

\section{Resumen}

Este estudio ofrece una propuesta lingüístico-comparativa entre el español y el siciliano. Mientras que se han realizado detalladas comparaciones entre lenguas romances, menos atención se le ha dado al siciliano, dado su carácter de dialecto del italiano. Según algunos lingüistas, el siciliano, como el sardo (hablado en Cerdeña), no se constituye como un dialecto, sino como una lengua independiente, que perdió su estatus de tal cuando el parlamento italiano decidió asignar una lengua oficial nacional. En esta investigación, independientemente de la interpretación del siciliano como lengua o como dialecto, se analizarán algunos de los aspectos fónicos, léxicos, ortográficos y morfológicos más sobresalientes que lo diferencian del español. Paralelamente, se establecen correspondencias con el italiano y el latín para entender mejor los procesos evolutivos del siciliano. Nuestro propósito reside en contribuir al canon analítico de estudios de dialectos y lenguas romances y en darle más visibilidad al siciliano, el cual cuenta con pocos estudios de esta índole.

Palabras clave: siciliano, español, variación lingüística del siciliano, historia de la lengua siciliana, dialecto, lengua.

\section{Comparative-linguistic study between sicilian and spanish languages}

\begin{abstract}
This study proposes a linguistic-comparative analysis of both Spanish and Sicilian. While numerous linguistic studies have been done on Romanic languages, very few deal with Sicilian, isolated as a dialect of Italian. According to some linguists, Sicilian, as well as Sardinian (spoken in Sardinia), is not a dialect but an independent language per se. It lost its language status when the Italian parliament decided to assign a national language. This research, independently of Sicilian being a language or a dialect, analyses the most important phonetic, lexical, spelling and morphological differences with his sister-language, Spanish. At the same time, some parallelisms with Italian and Latin will be presented to understand better the linguistic evolution of Sicilian. Therefore, our main goal lies on contributing to the analytical cannon of studies in both Romanic languages

* Investigación en lingüística comparativa entre el español y el siciliano. Portland State University.

** Doctora en lingüística española por la Universidad de Salamanca, España. Department of World Languages \& Literatures, Portland State University, USA, enunez@pdx.edu

***Master en Español. Department of World Languages \& Literatures, Portland State University, USA, raven@pdx.edu
\end{abstract}


Estudio lingüístico-comparativo del siciliano y el español / Eva Núñez, Raven Chakerian

and dialects, and on bringing up the importance of Sicilian, which has received very little attention in the field of linguistic.

Key words: Sicilian, Sicilian linguistic variation, history of Sicilian, dialect, language.

Recibido: 20-07-2011 Aceptado: 20-01-2012

\section{Introducción}

No cabe duda de que la historia geográfica y lingüística de la Península Itálica comparte muchas similitudes con la de la Península Ibérica. Ambas han desarrollado una trayectoria histórica semejante gracias a su proximidad en el Mediterráneo y a las continuas invasiones sufridas a lo largo de los siglos en el marco sur-europeo.

En la Península Ibérica se hablan en la actualidad cuatro lenguas romances: el portugués, el gallego, el catalán y el español, además de contar con numerosos dialectos como el andaluz, el extremeño, el aragonés, etc. Aunque Italia no presenta esta diversidad de lenguas, sí, en cambio, goza de una gran riqueza dialectal de norte a sur del país, por lo que se puede afirmar que la variedad lingǘstica caracteriza a los dos territorios. $\mathrm{El}$ amplio repertorio de variedades italianas (entre las que se encuentran el sardo, hablado en Cerdeña y considerado una lengua independiente romance, y el siciliano, hablado en la isla de Sicilia y considerado dialecto), dejó paso a la institucionalización del italiano como lengua oficial. La decisión del parlamento italiano de constituir el italiano como lengua oficial obedecía a criterios de unificación política y educativa.

Teniendo en cuenta que hoy en día se pueden encontrar infinidad de estudios comparativos lingüísticos de las lenguas romances, no deja de sorprender que el siciliano no haya acaparado la atención merecida. Por lo tanto, en este estudio, pretendemos ofrecer una propuesta comparativa de los aspectos fónicos, léxicos, ortográficos y morfológicos que más distinguen al siciliano del español y, por otro lado, contribuir al canon descriptivo de estudios lingüísticos en el grupo suritálicomediterráneo. Al mismo tiempo, se establecerán comparaciones con el latín y el italiano, para respaldar las diferencias y similitudes entre el español y siciliano. No es nuestra intención analizar la codificación lingüística del siciliano como dialecto del italiano o como lengua romance independiente, ya que este tema es polémico, sigue en el candelero y no es nuestro objetivo. Nuestra contribución se limita a una comparación

$+250$


sucinta entre lenguas y no a un estudio analítico del siciliano-lengua frente al siciliano-dialecto.

\section{Perfiles geográficos-lingüísticos del siciliano y del español}

La isla de Sicilia, parte del territorio italiano en el Mediterráneo, se ha constituido como un enclave militar y sociopolítico de suma importancia a lo largo de la historia. Por esta misma razón, ha sufrido múltiples conquistas y colonizaciones, parecidas a las que también fue sometida la Península Ibérica.

En su trayectoria histórica, estos dos pueblos coinciden en acaparar dos influencias esenciales para el desarrollo de su cultura y su lengua: la romana y la árabe. El impacto del latín y del árabe dejaron una profunda huella que sigue manifestándose en la actualidad.

Unos 3.000 años a. de C. predominaba la cultura íbera de la cual procede la denominación de ibérica atribuida a la península. Siglos después, aproximadamente en el 1.100 a. de C. llegaron los celtas y se mezclaron con las tribus autóctonas íberas, creando una nueva población: los celtíberos. A lo largo de ese periodo, establecieron colonias en el sur costero penínsular los griegos y fenicios, que constantemente surcaban las aguas del Mediterráneo en busca de metales y productos alimenticios. Estos no se mezclaron con los celtíberos, ya que no eran colonizadores, sino comerciantes marítimos, consecuentemente sus lenguas no se integraron en la trayectoria lingüística peninsular. Lo que hemos heredado del griego, se ha hecho a través del latín. El periodo celtíbero duró hasta la invasión romana en el 218 a. de C.

La llegada y la colonización romana, cuya administración permaneció en la península hasta el 409 d. de C., trajo consigo un cambio radical en los hábitos lingüísticos y en todas las facetas de la vida diaria. El latín se convirtió en la lengua predominante, desplazando a las otras lenguas naturales de la zona. El latín hablado, o vulgar, ya estaba predispuesto a cambiar de unas zonas a otras debido a la presencia de estas lenguas autóctonas, que, aunque se extinguieron, dejaron su pequeña marca sustratística. Con las invasiones germánicas en el siglo V, el latín no dejó de perder su vigencia, y fueron más bien los germanos los que se adaptaron al latín peninsular. A pesar del control territorial y político de los suevos, vándalos y alanos, estas tribus germánicas nunca impusieron ni propagaron su lengua. 
Con la llegada de los árabes en el año 711 de nuestra era, entra en la península otra influencia lingüística esencial en la constitución del español. El árabe contribuyó en gran medida a incrementar el caudal léxico del latín romanceado que se hablaba en el territorio peninsular. Con una presencia cultural de más de siete siglos, lograron influir en todos los aspectos de la sociedad.

Es el árabe, después del latín, el que más ha contribuido al léxico de la lengua española (Resnyck, 1989).

La historia de Sicilia se parece a la de España, por las numerosas invasiones y dominaciones foráneas que sufrió la isla. Como pasó con los comerciantes fenicios y griegos en la península, no todos los pueblos colonizadores que llegaron a la isla dejaron una huella lingüística. Su posición estratégica y los recursos mineros y agrícolas atrajeron a muchos invasores, comerciantes y navegantes, algunos de los cuales dejaron una impronta lingüística bastante variada.

Las tribus más antiguas que se reconocen como autóctonas de la isla son los sículos y los sicanos. Se cree que estos dos pueblos se establecieron en la isla unos 3000 años a. de C. y que convivían todos juntos, combinando sus tradiciones y su lengua. En el siglo VIII a. de C., llegaron los fenicios; al igual que en España, el fenicio no logró imponerse al habla de los lugareños, aunque los fenicios permanecieron casi setecientos años en la isla, hasta el 250 a. de C. Los griegos, comerciantes y marineros omnipresentes en el Mediterráneo, compartieron con los fenicios los recursos de la isla desde el 735 al 254 a. de C., ejerciendo una influencia palpable en la cultura y la lengua siciliana, de aquí las numerosas palabras de origen griego en siciliano. Después de los griegos, llegaron los romanos que se quedaron administrando la isla del 254 a. de C. hasta el 410 d. de C. Los romanos impusieron su lengua y su cultura del mismo modo que en la península. No obstante, los hablantes autóctonos continuaron utilizando su habla local y el griego. En esta época, la población de la isla se comunicaba de forma trilingüe, utilizando el latín y el griego como lenguas de comercio y el habla vernácula (Devoto, 1978).

Entre el 410 y el 535 fueron llegando las tribus germánicas (los vándalos y los ostrogodos), pero sin dejar mucha trayectoria lingüística al igual que en la península. Tampoco dejó huellas la dominación de los bizantinos desde el 535 hasta el 827, año en el cual se produce la entrada de los árabes en la isla. Los árabes permanecieron en Sicilia hasta el 1072 y, aunque su dominación no fue tan extensa como en la 
península, apenas más de dos siglos, sí lo fue su legado léxico. A continuación siguieron otros invasores de poca importancia lingüística, como los normandos (1072-1198), los suevos (1198-1266) y los francos con la dinastía angevina (1266-1282), (Maiden, 1995).

Finalmente, llegaron los españoles que se instalaron en la isla durante más de cinco siglos (1282-1860), sometiéndola a la Corona de Aragón, junto con el Reino de Nápoles y Cerdeña. Como consecuencia, el influjo de España aflora constantemente en el perfil cultural y lingüístico de Sicilia.

Tanto el siciliano como el español han recibido numerosas influencias lingüísticas a lo largo de los siglos, unas de mayor y otras de menor envergadura, sin embargo, el latín y el árabe resaltan como las que más han dejado (Sucato, 1975); lo que resulta lógico siendo el siciliano una lengua neolatina. De las 250.000 voces con las que cuenta el siciliano, la mayoría provienen del latín, griego o árabe.

Sicilia pasó a formar parte de Italia en el siglo XIX, desde entonces su historia ha estado vinculada estrechamente a la de Italia y no a la de España. Hoy en día La Región Autónoma Siciliana tiene su propio gobierno y parlamento regional.

\subsection{La ortografía siciliana y la variación lingüística de Sicilia}

Hasta el año 1800, la ortografía siciliana siguió un patrón estrictamente etimológico. Sin embargo, esta correspondencia entre étimo y grafía se ha alterado en los últimos dos siglos, lo que ha resultado en una falta de coherencia ortográfica. Una misma palabra puede aparecer escrita de distintas maneras. La realidad es que no hay un consenso sobre seguir una propuesta ortográfica de corte etimológico u otra de corte fonético.

La mayoría de los autores abogan por mantener la ortografía del siciliano, según una pauta etimologizante, para dar mayor dignidad literaria o prestigio a la variedad siciliana. Paralelamente, la confusión entre "b" y "v" en español y su representación ortográfica tomó un derrotero similar, cuando la RAE acudió a la fuente etimológica para representarlas en la escritura.

En general, en este estudio se seguirán las pautas de Antonella Fortuna en Grammatica siciliana: principali regole grammaticali, fonetiche e grafiche (comparate tra i vari dialetti siciliani). Fortuna proporciona variaciones dialectales del siciliano en diversas zonas de la isla. Esta investigación no 
pretende ser exhaustiva en cuanto al análisis de las variaciones lingüísticas en la isla, sino que analiza aquellos ejemplos más destacados que diferencian al siciliano del español. Es necesario recalcar que el siciliano moderno presenta numerosos italianismos por los lazos políticos que mantiene con el gobierno central de la República; además los jóvenes tienden a preferir el italiano y se resisten a hablar siciliano. Otros isleños hablan una mezcla entre italiano y siciliano; son los ancianos los que siguen con sus tradiciones y hablando siciliano.

\subsection{El grupo itálico del siciliano y del español}

Se sigue asumiendo, en términos generales, que tanto el español como el siciliano proceden del mismo grupo indoeuropeo itálico, derivados de la misma lengua-madre: el latín vulgar. Resulta obvio mencionar que el latín ha sido el esqueleto original en la formación del español, el siciliano y el italiano, por lo cual estas tres derivaciones romances cuentan y comparten un alto contenido de vocablos y de aspectos morfosintácticos, procedentes del latín hablado o vulgar.

Mientras que no se duda de la derivación del español de su lenguamadre, el latín, cuando se trata del siciliano, algunos lingüistas ponen en entredicho tal afiliación lingüística y llegan a promover teorías un poco extremistas. Estos abogan por la idea de que el siciliano viene de otra lengua no latina, el protosiciliano, como origen primordial, aunque no descartan la gran aportación latina. Con respecto a la existencia o no del protosiciliano, carecemos de un consenso general y de información contundente documentada: para algunos académicos, se trata de un dialecto originario del latín primitivo, mientras que, para otros, se trata de una lengua independiente, hermana de la latina, que también proviene del grupo indoeuropeo. Esta misma dialogía se traspasa a la interpretación del siciliano: ¿cuál es el estatus lingüístico del siciliano moderno, dialecto o lengua?

Aquellos estudiosos que lo consideran una lengua recurren al protosiciliano, definiéndolo como lengua independiente del latín, no derivada de ésta. Entre estos se encuentra el lingüista Privitera que defiende y cuestiona su teoría en el volumen titulado Sicilian: The Oldest Romance Language. También Sucato sigue esta tendencia y, además, propone audazmente que el latín tiene sus orígenes en el protosiciliano en su libro La lingua siciliana: origine e storia. Para más información sobre el protosiciliano, léase a Mazzola (1976). 
Otros expertos han destacado que el siciliano mantiene algunos latinismos que no pervivieron en los demás dialectos romances. De hecho, Bonfante (1986) compila una lista de estos latinismos en La continuità del latino in Sicilia. Algunos de los elementos mencionados en esta lista, incluyen la forma verbal que proviene del perfecto latino y la tendencia sintáctica de colocar el verbo al final del enunciado.

En el presente estudio, se opta por la opinión más generalizada de aceptar el siciliano como variante romance derivada del latín vulgar y como dialecto del italiano sureño. Es decir, no se lo analiza como una lengua per se.

\section{Comparaciones léxicas y fonéticas}

A continuación, siguen algunas comparaciones léxico-etimológicas entre el español, el siciliano, el italiano y su origen latino. En algunos casos, el siciliano ha sufrido un desarrollo fonético muy parecido al del español; en otros, sigue al italiano. Además, hay casos donde la evolución del siciliano no se asemeja a ninguna otra lengua romance.

Para esta sección se analizan los grupos consonánticos y vocálicos que mayores cambios presentaron en la evolución del siciliano, italiano y español. Se han elegido porque son los que más variación lingüística presentan con respecto a su origen latino.

\subsection{El grupo consonántico latino <-ll->}

Tanto en español como en italiano, en posición interior de palabra, la grafía doble latina <-LL-> se suele conservar, no obstante, la pronunciación latina de $<-\mathrm{LL}->$, como $<\mathrm{l}>$ doble o prolongada, se conserva en italiano pero no en español. En español la <-LL-> latina palatalizó en [y] (yeísmo) y [K] (lleísmo).

En siciliano, en cambio se produjo un resultado gráfico y fonético completamente diferente, la grafía $<1 l>$ dio $<\mathrm{dd}>$, pronunciada como cacuminal [ḍ̣] .

\begin{tabular}{|c|c|c|c|}
\hline Latín & Español & Italiano & Siciliano \\
\hline CABALLUM & caballo & cavallo & cavaddu \\
\hline GALLUM & gallo & gallo & iaddu, jaddu \\
\hline PATELLAM & paella & padella* & paredda, padedda \\
\hline COLLUM & cuello & collo & coddu \\
\hline BELLA & bella & bella & bedda, bbedda \\
\hline
\end{tabular}

* padella en italiano es un tipo de sartén, como la paellera española. 


\subsection{El grupo consonántico latino <-gn->}

El grupo consonántico <-GN-> del latín generalmente palatalizó en español, italiano y siciliano, dando el sonido [n], representado gráficamente en español con la eñe $<\tilde{n}>$. En italiano y siciliano se mantuvo la ortografía latina; sin embargo, en ninguna de las tres variantes romances el grupo $<$ gn> mantuvo la pronunciación latina de [gn].

Caso de especial interés es la voz latina Lignum, ya que la palabra singular del español y el siciliano procede del sustantivo neutro plural latino y no del singular. El neutro plural en latín terminaba en -A, lo que a veces causó confusiones con la evolución del género de algunas palabras que pasaron a ser femeninas aunque en latín eran de género neutro. En italiano, en cambio, el sustantivo neutro singular pasó a ser el sustantivo masculino singular.

\begin{tabular}{|c|c|c|c|}
\hline Latín & Español & Italiano & Siciliano \\
\hline $\begin{array}{c}\text { LIGNUM } \\
\text { LIGNAM (pl.) }\end{array}$ & $\begin{array}{c}\text { leño } \\
\text { leña }\end{array}$ & legno & ligna \\
\hline COGNATUM & cuñado & cognato & cugnatu \\
\hline INSIGNARE & enseñar & insegnare & insignari \\
\hline AGNELLUM & (cordero) & agnello* & agneddu, agniddu* \\
\hline PIGNUM & peño** & pegno & pignu \\
\hline
\end{tabular}

*cordero en español, entre paréntesis para indicar otro origen etimológico.

** peño antiguamente lo que se da o se deja en prenda (palabras derivadas son empeño, desempeño)

\subsection{El grupo consonántico latino <-ct->}

En el caso del grupo consonántico latino <-CT->, el siciliano siguió la misma evolución que el italiano: la $\langle\mathrm{c}\rangle$ se asimiló a la $\langle\mathrm{t}\rangle$, con lo que se dio la geminación de $<\mathrm{tt}>$ que se pronuncia como una única consonante prolongada, como si se tratara de [t] más [t]. En español en cambio hubo palatalización de la consonante dental por lo que la combinación $<c t>$ pasó generalmente a $<\mathrm{ch}>$, pronunciada como [tg] en español estándar.

\begin{tabular}{|c|c|c|c|}
\hline Latín & Español & Italiano & Siciliano \\
\hline PECTUM & pecho & petto & pettu \\
\hline FACTUM & hecho & fatto & fattu \\
\hline NOCTEM & noche & notte & notti \\
\hline FRUCTUM & fruto & frutto & fruttu \\
\hline LACTUCAM & lechuga & lattuga & lattuca \\
\hline
\end{tabular}

$\longrightarrow$




\subsection{Los grupos latinos $<-\mathrm{ti}->\mathrm{y}<-\mathrm{ci}->$}

Los grupos latinos de interior de palabra <-TI-> y <-CI-> se asibilaron en una etapa intermedia como [ts] o [dz] hasta llegar a la interdentalización en [ $[\theta]$ en el español del centro-norte y en [s] en el andaluz. En italiano y en siciliano resultaron dos soluciones: el grupo dentoalveolar [ts] representado con la grafía <z>, como en forza [fórtsa]; y la consonante palatal $[\mathrm{t}]$, representada gráficamente como $\langle\mathrm{cc}\rangle$, por ejemplo en minaccia [minátfa].

Las evoluciones de estos grupos latinos resultaron bastante homogéneas en sus derivaciones italiana y siciliana, aunque hay algún caso particular como el de cuminitiare donde acabó en una sola $<\mathrm{c}>$. En esta última palabra la $<c>$ no queda en posición intervocálica, por lo que la posibilidad de $<\mathrm{cc}>$ no existe, aunque la pronunciación siga la norma indicada, dando la africada.

\begin{tabular}{|c|c|c|c|}
\hline Latín & Español & Italiano & Siciliano \\
\hline FORTIAM & fuerza & forza & forza [fórtsa] \\
\hline PETTIAM & pieza & pezza & pezza [pétsa] \\
\hline MINACIAM & amenaza & minaccia & minaccia [minátfa] \\
\hline CUMINITIARE & comenzar & cominciare & accuminciari [akumintfári] \\
\hline
\end{tabular}

\subsection{Los grupos latinos -cul-, -tul-, -gul- y -ly-}

En español las combinaciones <-CUL->, <-TUL->, <-GUL-> y <-LY-> se palatalizaron primero y después se velarizaron dando como resultado la consonante velar $[\mathrm{x}]$, representada por la grafía $<\mathrm{j}>$ en la mayoría de los casos.

En italiano, a veces estos grupos mantienen la palatalización pero sin velarización, pronunciándose como una consonante lateral palatal $[K]$ y escribiéndose como $<$ gli $>$. Otras veces estos grupos se velarizan en $[\mathrm{k}]$, representándose en la escritura como <cch>.

En siciliano los resultados se presentan de forma irregular: normalmente se pronuncian como una consonante velar sonora [g], escrita como $<$ ggh $>$ o como velar sorda [k], con las grafías $<\mathrm{cch}\rangle$. A veces no hay síncopa de la vocal $<\mathrm{u}>$ en el grupo $<\mathrm{tul}>$ y la $<\mathrm{t}>$ se asibila dando como resultado el sonido [ts], escrito como <zz>; por lo que el resultado final <zzul> es pronunciado como [tsul].

Fonéticamente, tanto en italiano como en siciliano, estos grupos pueden dar como resultados consonantes largas del tipo [kk] en italiano; y $[\mathrm{kk}]$ y $[\mathrm{gg}]$ en siciliano. 
Estudio lingüístico-comparativo del siciliano y el español / Eva Núñez, Raven Chakerian

\begin{tabular}{|c|c|c|c|}
\hline Latín & Español & Italiano & Siciliano \\
\hline CONICULUM & conejo & coniglio & cunigghiu \\
\hline OCULUM & ojo & occhio & occhiu \\
\hline VETULUM & viejo & vecchio & vecchiu \\
\hline ARROTULARE & arrojar & arrotolare & arruzzulari \\
\hline COAGULARE & cuajar, coagular* & cagliare, coagulare* & cuagulari, quagghiari \\
\hline PALEAM & paja & paglia & pagghia \\
\hline FILIAM & hija & figlia & figghia \\
\hline UNGULAM & uña & unghia & ugna, unghia** \\
\hline
\end{tabular}

*Coagulare del latín se mantuvo como cultismo "coagular" en español y coagulare en italiano. En siciliano solo dio cuagulari.

**Ungulam resultó en ugna y unghia según sea el dialecto siciliano.

\subsection{El grupo consonántico pl- inicial}

El grupo consonántico inicial <PL-> del latín se palatalizó en español convirtiéndose en el sonido lateral palatal $[K]$ o fricativo palatal [y] (yeísmo), escrito como $<$ ll $>$. En italiano se vocaliza la consonante lateral en $<\mathrm{pi}>$; y en siciliano, además de vocalizarse la lateral, se velariza la oclusiva inicial en [ki], en la ortografía <chi>. Compárense los siguientes ejemplos.

\begin{tabular}{|l|l|l|l|}
\hline Latín & Español & Italiano & Siciliano \\
\hline PLOVERE & llover & piovere & chiuviri [kjúviri] \\
\hline PLUMBUM & plomo & piombo & chiummu [kjúmmu] \\
\hline PLUS & plus** & piu* & chiu [kjú]* \\
\hline PLANUM & llano, plano & piano & chianu [kjánu], pianu \\
\hline PLENUM & lleno, pleno & pieno & chiinu [kínu] \\
\hline
\end{tabular}

*piu y chiu equivalen al adverbio "más" procedente de otro étimo, (ejemplo: chiu granni en siciliano = más grande en español)

** plus existe en español como forma invariable aunque según la RAE su significado se relaciona al "más" latino sólo de forma parcial.

\subsection{La consonante intervocálica latina $<-\mathrm{x}->$}

En latín la grafía <-X-> se pronunciaba como [ks]; esta combinación evolucionó de varias maneras en las lenguas romances. En español se transformó en la consonante velar fricativa sorda [x], representada en la escritura con $\langle j\rangle y<g\rangle$. En italiano y en siciliano se derivó principalmente en una "s" larga o pronunciada [ss], escrita como <ss $>$ reproduciendo fielmente la pronunciación.

A continuación, se enuncia el caso de Fraxinum como ejemplo de una evolución en español similar a la del italiano y el siciliano. 


\begin{tabular}{|c|c|c|c|}
\hline Latín & Español & Italiano & Siciliano \\
\hline TEXERE & tejer & tessere & tessiri \\
\hline CONDUXI & conduje & condussi & cundussi \\
\hline FRAXINUM & fresno & frassino & frassinu \\
\hline
\end{tabular}

A veces la $<x>$ intervocálica se transformó en una consonante alveopalatal fricativa sorda reduplicada [ $\left.\iint\right]$ tanto en italiano como en siciliano en lugar de dar la evolución más común de [ss]. Nótese que las grafías $<$ sC $>$ se pronuncian como [J]] en italiano y en siciliano.

\begin{tabular}{|c|c|c|c|}
\hline Latín & Español & Italiano & Siciliano \\
\hline AXILLAM & axila & ascella & 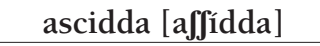 \\
\hline COXAM & (muslo)* & coscia & cuscia [cúffja], coscia \\
\hline EXIT & $(\text { salida })^{*}$ & esce & nesci [né $\left.\int \mathbf{i}\right]$ \\
\hline
\end{tabular}

* entre paréntesis para indicar otro origen etimológico.

\subsection{La $<-u>$ final latina}

La vocal final latina <-u> se convierte en <o> en español y en italiano, marcando la diferencia morfológica de género masculino (en la mayoría de los casos); en siciliano, en cambio, se mantiene como <-u>.

\begin{tabular}{|c|c|c|c|}
\hline Latín & Español & Italiano & Siciliano \\
\hline VICINUM & vecino & vicino* & vicinu* \\
\hline FICUM & higo & fico & ficu \\
\hline CAPUT & cabo & capo & capu \\
\hline CAELUM & cielo & cielo* & celu* \\
\hline SPECULUM & espejo & specchio & specchiu \\
\hline
\end{tabular}

* La $<\mathrm{c}>$ ante $<\mathrm{e}>\mathrm{o}<\mathrm{i}>$ se pronuncia como consonante palatal $[\mathrm{t}]$ en italiano y siciliano

El sistema vocálico del siciliano, así como del español y del catalán, se presenta bastante simplificado, en comparación con otras lenguas romances donde se recogen más diferencias vocálicas, en cuanto a la articulación y nasalidad (como ocurre con el portugués o el francés). El siciliano y el español mantienen una correspondencia bastante análoga en cuanto a las vocales, cinco en total; por lo que se refiere a los diptongos, el siciliano presenta vocales simples $<$ e $>$ y $<0>$ donde el español presenta $<$ ie $>$ y $<$ ue $>$ (Tornatore, 2003), lo que también ocurre en otras variedades romances como el italiano y portugués. Compárense las palabras puente, fiesta y yegua: 
Estudio lingüístico-comparativo del siciliano y el español / Eva Núñez, Raven Chakerian

$\begin{array}{lllllll}\text { latín } & \text { siciliano } & \text { italiano } & \text { español } & \text { portugués } & \text { rumano catalán francés } \\ \text { PŏNTEM ponte } & \text { ponte } & \text { puente } & \text { ponte } & \text { pod pont pont } \\ \text { FĚSTAM festa } & \text { festa } & \text { fiesta festa } & \text { (petrecere)* festa féte } \\ \text { ĚQUAM } & (\text { cavadda })^{*} & \text { (cavalla)* yegua } & \text { égua } & \text { iapă } & \text { egua, euga (jument)* }\end{array}$

* entre paréntesis indica otro origen etimológico

\subsection{La /i/ siciliana y sus alófonos}

Lo más interesante de la /i/reside en su variada representación ortográfica. El fonema vocálico /i/ suele resultar problemático en la ortografía siciliana dadas las variaciones de pronunciación que conlleva. Este fonema cuenta con tres alófonos o variantes en la lengua hablada. A lo largo de la historia literaria siciliana la /i/ se ha visto escrita tanto con la grafía $<\mathrm{i}>$ como con la $<j>$ con poca consistencia y regularidad. En Grammatica siciliana, Salvatore Camilleri propone normalizar la /i/ en la ortografía siciliana y sugiere utilizar la letra $<j>$ para la semiconsonante [j] (antes de vocal) y la grafía <i> para la vocal [i] y la semivocal [i] (después de vocal).

Entre consonantes la /i/ produce [i] como se ejemplifica en la palabra virità [viritá]. En posición intervocálica, en cambio, el alófono que resulta es la semivocal [i] como en la palabra maiu [maiu]. Cuando la /i/ no es intervocálica, por ejemplo a principio de enunciado o de palabra, se considera una semiconsonante [j] como en la palabra jornu [jórnu]. La semiconsonante al principio de palabra es más común en siciliano que en español o italiano. En lugar de las consonantes al principio de palabra $[\mathrm{dd} z],[\mathrm{d}]$ y $[\mathrm{g}]$ del italiano y $[\mathrm{x}],[\mathrm{d}]$ y $[\mathrm{g}]$ del español, en siciliano aparece la semiconsonante [j] como se puede ver en la siguiente tabla, donde se recogen las dos variaciones ortográficas posibles en siciliano.

\begin{tabular}{|c|c|c|c|}
\hline Latín & Español & Italiano & Siciliano \\
\hline DIURNuS & jornada & giornata [ddzornáta] & jornata, iornata [jornáta] \\
\hline (gard)* & jardín & giardino [ddzardíno] & jardinu, iardinu [jardínu] \\
\hline DIGITUM & dedo & dito [díto] & jitu, iitu [jítu] \\
\hline GALLum & gallo & gallo [gállo] & jaddu, iaddu [jáddu] \\
\hline
\end{tabular}

* del franco gard, cercado; entre paréntesis para indicar otro origen etimológico.

\subsection{Palabras latinas que mantienen su valor semántico en siciliano}

Desde el punto de vista léxico-semántico, se pueden anotar algunas diferenciaciones. En siciliano aparecen voces que mantienen el mismo significado que tuvieron en latín, mientras que en italiano o español

260


desaparecieron o modificaron parcialmente su significado, como por ejemplo muscaloru, culostra, o cambiaron como forficia (tijeras), nolitu (capricho) y requia (paz). Algunas de ellas, no sobrevivieron en todos los dialectos sicilianos y se reemplazaron por palabras italianas. Véase el recuadro a continuación.

\begin{tabular}{|c|c|c|c|}
\hline Latín & Español & Italiano & Siciliano \\
\hline FORFEX & (tijeras) & forbici & forficia [forfítfa], fovvici \\
\hline NOLITUM & (capricho) & (capriccio) & nolitu \\
\hline MUSCARIUM & & & muscaloru* \\
\hline COLOSTRUM & calostro** & colostro** & culostra $* * *$ \\
\hline HODIE EST ANNUS & año (pasado) & anno (scorso) & oggellannu [odzelánu] \\
\hline $\begin{array}{c}\text { A + CERTUM }>\text { ACERTARE } \\
\text { ADDIVINARE }\end{array}$ & $\begin{array}{l}\text { acertar } \\
\text { adivinar }\end{array}$ & $\begin{array}{c}\text { accertare } \\
\text { indovinare }\end{array}$ & $\begin{array}{c}\text { nzertari [nzertári] } \\
\text { nzttari }\end{array}$ \\
\hline REQUIEM & (paz) & (pace) & requia [rékwia], paci \\
\hline
\end{tabular}

*El muscaloru es un matamoscas en forma de abanico.

** El calostro según la RAE es la primera leche que da la hembra después de parir.

También tiene este significado colostro en italiano y lo tenía en latín colostrum.

*** Culostra se usa para describir a un animal que ha parido recientemente.

Como caso destacado, se señala la palabra latina Forfex que evolucionó distintantemente con un cambio morfológico de número, dando plural en italiano como sustantivo femenino le forbici y singular en siciliano la forficia.

\section{La influencia árabe}

Los árabes habitaron casi 300 años en Sicilia y unos 800 en la Península Ibérica. Esta larga presencia permitió que dejaran una huella lingüística importante en el caudal léxico, tanto del español como del siciliano. Esta herencia se manifiesta sobre todo en la toponomía y en los vocablos para la comida, las herramientas, los productos agrícolas, plantas y en otros tantos aspectos de la actividad diaria.

Si tenemos en cuenta la toponimia, por ejemplo, numerosos pueblos sicilianos llevan nombres árabes: Palermo, Ficarazzi, Cefelá, Zaffarana y Aspra. Otros pueblos se denominan haciendo referencia a un producto agrícola cultivado en la zona o que se comercializaba. Por ejemplo: Zaffarana y Safarella se refieren a la especia del azafrán, za'faran en árabe, que era y sigue siendo un ingrediente frecuente en la cocina árabe. Calaseibetta recibe su nombre del árabe qal'ah-sibit, "lugar del eneldo". Otros topónimos relacionados con la agricultura son Raciura, proveniente del árabe scuirah o "lugar donde abunda el agua", Garaffa que significa 
"molino de agua" en árabe y Fastuca que proviene del árabe fustuq o pistacho. Los pueblos Sabuccia y Zaituni se derivan de la palabra aceituna en árabe, que como ya se sabe, también se mantuvo en español junto con su correspondiente latino oliva (Guerriero, 1998).

Se cree que los nombres de algunos pueblos como por ejemplo Indulsi, al-andalus en árabe, llegaron al siciliano por mediación de los colonizadores españoles que, a su vez, ya lo habían adquirido del árabe.

También en el nombre de las calles se observa la influencia árabe, como la calle Muschita en Palermo cuyo nombre proviene de mesged, mezquita. En la misma ciudad existe un castillo llamado Kalsa, del árabe kalisah', la pura o la elegida.

Del mismo modo, en España, los árabes contribuyeron enormemente a la toponimía peninsular, no solo en las zonas sur y este bajo un extenso poder musulmán, sino también en zonas de la meseta y del noroeste. Son topónimos árabes Albacete, Alcalá, Algarbe, Alhambra, Alicante, Almería, Almodóvar, Benalmádena, Benicásim, Calatayud, Guadalcanal, Guadalajara, Guadalén, Guadalquivir, Guadalupe, Guadarrama, Gibraltar, La Mancha, Medina, Medinaceli y muchos otros (García Sánchez, 2007). Estas denominaciones de lugares suelen ser descriptivas, como ejemplifican los derivados de guada- de wad-al, el río, y los del componente ben-o beni que indican "hijos de".

Palabras de uso cotidiano en la casa como almohada del español y cadda del siciliano, vienen de la palabra árabe hoadodo. La interjección española ;hala! viene de ya allah; mientras que la siciliana asara, ipor dios!, de ya hoasra.

Otras palabras de uso común en siciliano tomadas del árabe son mattaffu, lleno, de mataff; mazzara, objeto pesado, de mas'sara; sceccu, asno, de sheik; tabutu, cofre, de tabut; y burnia, un jarrón de vidrio, del árabe burnía.

De la misma forma, el léxico de la cocina se vio influenciado por los árabes, que introdujeron comidas nuevas en las regiones de su imperio, por lo que se adquirieron nuevas voces para plantas, verduras, frutos, especias y platos. En siciliano se usa caccioffulu, alcachofas, del árabe kharshu'f; addumi, dátil, de daum; butáraca, los ovarios de un pescado seco que se come, de butuarih; cacicia, un tipo de betaraba amarilla, de huaseise; caleca, ciruela, de hawaiha; calia, garbanzos tostados, del árabe haliah (Privitera, 2004).

$+262$


En español abunda el vocabulario de procedencia árabe para referirse a productos alimenticios como aceite, aceitunas, acelga, arroz, alcachofas, ajonjolí, albaricoque, albóndigas, almidón, almizcle, alcohol, berenjena, bellota, café, naranjas, hachís, judía, limón, sandía, toronja, zanahoria, etc.; en muchas ocasiones estos vocablos son cognados en español y siciliano.

A diferencia del español (también del portugués y del catalán), el siciliano no presenta una incidencia alta de sustantivos con el artículo árabe al aglutinado. Los estudios románicos de Colón han analizado las diferencias en la incorporación de arabismos en el castellano, el catalán y el siciliano, comparando las palabras que empiezan por a(l)del diccionario de Nebrija. Sus resultados reflejan que el castellano es el idioma que tiene más arabismos de estos tres. El catalán, a primeros del siglo XVI, tenía un cincuenta y tres por ciento de los arabismos del castellano y el siciliano, veinte y dos. Actualmente solo un dos por ciento del léxico catalán son arabismos, frente al ocho por ciento del castellano (Colón, 1976).

Tanto el catalán como el siciliano, en la actualidad, presentan un número reducido de arabismos (si lo comparamos con el del español), lo que se explica perfectamente por la situación geográfica al margen del Al-Andalus y, en el caso del catalán, además por la Reconquista más temprana. Las pocas generaciones que separan la conquista árabe del AlAndalus (711) y la de Sicilia (827) no determinan esta variación lingüística, más intensa en la península y reducida en la isla ${ }^{1}$. Una explicación actualizada de esta diferencia reside en afirmar que los arabismos (fácilmente identificables por la aglutinación del artículo al- o a- entre otros

1 La mayoría de los críticos defienden el origen beréber del artículo árabe al- en el léxico español. Elcock (1960) atribuye la aglutinación de al- a la inexistencia de artículo en las hablas bereberes, lo cual impidió la identificación y segmentación del artículo en estas hablas. Lüdtke (1968) amplió esta tesis, aclarando que las lenguas africanas orientales y de Asia carecían de artículo aglutinado; en cambio las de África occidental y las bereberes sí. Corriente (1999) sigue y completa las tesis de Elcock y Lüdtke, concretizando la particularidad del árabe berberizado, una especie de árabe criollizado o pidginizado, con uso abusivo del artículo, que se difundió entre los nativos hispanovisigodos. Después de un largo proceso de nivelación de rasgos dialectales y de formación, este árabe andalusí, procedente de bereberes bilingües, quedó sumamente diferenciado con respecto a otras variedades como las suditálicas, incluyendo Sicilia. Noll (2006) difiere de las posturas anteriores y defiende que el abuso del artículo del árabe berberizado no explica la aglutinación característica de los iberorrománicos sino que se debe a un contexto bilingüe activo. Es decir, para Noll el impacto lingüístico beréber no viene condicionado por la mayor o menor cantidad de palabras con aglutinación, sino en la situación bilingüe de las culturas en cuestión: "resulta difícil reducir al mínimo la influencia beréber en Sicilia por falta de aglutinación, mientras que se la pone de relieve en al-Andalus y en el Magreb occidental" (2006). 
aspectos morfológicos), provienen de los préstamos en un medio bilingüe mozárabe activo, no del contacto habitual de lenguas (Noll, 2006).

Los arabismos, tanto en español como en siciliano, abarcan todos los campos de la actividad humana, y según Lapesa suponen, después del latín, el caudal léxico más importante del español (Lapesa, 1995). Representan el ocho por ciento del vocabulario total español y se calcula que pueden llegar a ser unas cuatro mil palabras (Cano, 1999).

Dejamos sin comentar muchas otras palabras del árabe incorporadas al español y al siciliano, ya que este trabajo no pretende ser exhaustivo de la herencia léxica de la lengua árabe. No obstante, hay que resaltar que el patrimonio lingüístico árabe fue esencial en la formación, tanto del español como del siciliano. Su huella sigue indeleble.

\section{Comparaciones morfosintácticas}

Los españoles ocuparon Sicilia más de cinco siglos, desde finales del siglo XIII hasta mediados del siglo IX. Aunque durante este periodo no reinaron en la isla de forma continua, sí estuvieron en contacto directo con sus habitantes por razones político-mercantiles, ejerciendo una influencia cultural y lingüística sobresaliente y única. La huella lingüística del español se manifiesta sobre todo en la terminología náutica, militar y comercial.

Sicilia, junto con Rodas, eran enclaves importantes para el comercio del oeste del Mediterráneo, situadas en una de las rutas más codiciadas para el tráfico portuario. Las naves de España, al pasar por Sicilia, recogían granos y otros productos sicilianos, y otras veces se detenían en Rodas para cargar mercancias catalanas almacenadas allí. Durante el siglo XV, solamente Sicilia absorbía una cuarta parte del movimiento comercial que salía de Barcelona (Tornatore, 2003).

Este contacto directo y la proximidad geográfica entre la isla y el territorio catalán, posibilitó la incorporación de catalanismos en el siciliano, sobre todo en lo que se refiere al vocabulario descriptivo del mar, a lo naútico, el clima y todo lo relacionado con la navegación. Algunas voces sicilianas procedentes del catalán son avvistari (avistar), 'ncagghiari (encallar), funnali (hondonada), ammurrari, ammugghiari (amarrar), pavisata (arsenal), suttaventu (sotaviento), venticciolu, venticedda (viento ligero, ventijol en catalán), baccalaru (bacalao), anciova (anchoa), alaccia, aliccia (una especie de sardina, alatxa en catalán), traficari, trafichiari (comerciar), proffitu (ganancia), scottu (tela de algodón), carricaturi

$+264$


(cargadero), chiavitteri (cerrajero), michiletti (policías), bagaseri (vago mujeriego), cantunera (ramera) entre muchas otras ${ }^{2}$.

Los préstamos hispánicos en el siciliano resultan innumerables, por el contacto largo y profundo entre estas dos culturas. Las lenguas castellana y catalana jugaron un papel primordial en el gobierno, la milicia y el comercio sicilianos, posibilitando la infiltración idiomática en la lengua receptora.

La influencia lingüística del español en el siciliano se puede apreciar a distintos niveles. A continuación, se examinarán algunos casos particulares en la morfología y en la sintaxis.

En numerosas ocasiones se adopta la palabra española, pero con la terminación siciliana; así, se encuentran verbos españoles con las terminaciones de los verbos en infinitivo del siciliano. Por ejemplo, en appruvicciari, aprovechar, se observa que al verbo español se le añade la terminación del siciliano <-ari>; en la pronunciación se alarga la <p> en $<p p>$, bastante frecuente en siciliano; y las vocales "o" y "e" se cierran un grado en "u" e "i". También se mantiene la grafía $<v>$, procedente de la -F- latina como en español, aunque la pronunciación difiere en ambas lenguas, realizándose como bilabial en español [ $\beta$ ] y como labiodental en siciliano [v]. Así la raíz latina de este verbo, PROFECTum, evolucionó a provecho en español y profito en italiano (verbo approfittare).

En algunos casos, las palabras heredadas del español en siciliano se parecen a sus cognados italianos dado que provienen de la misma raíz latina. En otros, el vocablo siciliano heredado del español difiere del italiano aunque tengan el mismo valor semántico, es decir, las palabras sicilianas y españolas no comparten la misma raíz con la italiana, no son cognados, aunque signifiquen lo mismo. Esto se ve, por ejemplo, con la voz manta en español y siciliano equivalente a coperta en italiano.

\begin{tabular}{|c|c|c|}
\hline Español & Italiano & Siciliano \\
\hline aprovechar & approfittare & appruvicciari, apprufittari \\
\hline brazo & bracchio & vrazzu \\
\hline cita* $^{*}$ & fidanzata* & zita* $^{*}$ \\
\hline emborrachar & ubriacare & abburracciari, 'mbriacari \\
\hline ensayar & provare & 'nsaiari \\
\hline estropearse & inciampare & astruppiarisi \\
\hline farfante & bugiardo & farfante \\
\hline
\end{tabular}

2 Este léxico procede de Tornatore pp. 71, 78, 91, 105, 119, 140, 150, 153, 200, 239 y 240. Para más información sobre la influencia del catalán en el siciliano, léase a Tornatore (2003). 
Estudio lingüístico-comparativo del siciliano y el español / Eva Núñez, Raven Chakerian

\begin{tabular}{|c|c|c|}
\hline lástima & seccatura & lastima \\
\hline manta & coperta & manta \\
\hline padrastro & patrigno & parrastru, patrastru \\
\hline pasear & passeggiare & passiari \\
\hline
\end{tabular}

* fidanzata, "novia", es equivalente semántico para el siciliano zita, "prometida"; el español cita aparece aquí por ser el origen de la voz siciliana.

\subsection{Diferencias morfológicas de artículos y pronombres}

En latín no existían los artículos definidos (ni indefinidos), ni tampoco los pronombres personales de tercera persona para el objeto directo e indirecto, lo(s), la(s), le y se. La presencia de estos, tanto en español como en siciliano e italiano, se debe a la evolución del demostrativo iLle en sus casos correspondientes. Los resultados en español presentan un sistema más sencillo en cuanto al siciliano y al italiano.

En español, la pérdida de tonicidad y el carácter proclítico (delante del sustantivo) de ille provocaron la elisión de la vocal inicial a veces, y otras, de la vocal final, con la consecuente reducción de -LL- > -1(Núñez, 2012).

\begin{tabular}{|c|c|c|c|}
\hline & Artículos definidos & & ĬLLE $>$ elle $>$ ele $>$ el \\
\hline nom. masc. & 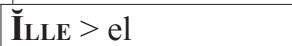 & \multirow{3}{*}{$\stackrel{00}{\tilde{\omega}}$} & ILLLA $>$ ella $>$ ela $>$ la \\
\hline nom. fem. & $\breve{\mathbf{I}}_{\mathbf{L L A}}>\mathrm{la}$, el & & $>$ ella $>$ ela $>$ el \\
\hline nom. neutr. & $\breve{\mathbf{I}}_{\text {LLUD }}>$ 10 & & $\breve{I L L U D}_{\text {L }}>$ illo $>$ ello $>$ elo $>$ lo \\
\hline acus. masc. & $\breve{\mathbf{I}}_{\mathbf{L L}} \overline{\mathbf{O}}_{\mathrm{S}}>\operatorname{los}$ & \multirow{2}{*}{$\frac{\dot{\Xi}}{2}$} & 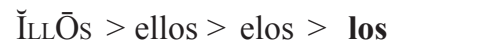 \\
\hline acus. fem. & $\breve{\mathbf{I}}_{\mathbf{L L A}} \overline{\mathbf{A}} \mathbf{S}>$ las & & $\breve{I L L}_{\mathrm{L}} \overline{\mathrm{S}}>$ ellas $>$ elas $>$ las \\
\hline
\end{tabular}

En italiano y siciliano la consonante doble de ILLE también se reduce, pero las vocales finales tienden a perderse; en el caso del siciliano, hasta la consonante doble puede desaparecer.

\begin{tabular}{|c|c|c|c|c|c|}
\hline \multicolumn{5}{|c|}{ Artículos definidos } & \\
\hline & Latín & Español & Italiano & Siciliano & \\
\hline masc. & $\breve{I}_{\text {LLE }}>$ & el (el perro) & il, lo, l' (il cane) & lu, 'u, l' ('u cáni) & \\
\hline fem. & $\breve{I L L A}_{\text {LLA }}$ & $\begin{array}{l}\text { la (la mesa) } \\
\text { el (agua) }\end{array}$ & $\begin{array}{c}\text { la (la tavola) } \\
\text { l' (l'acqua) }\end{array}$ & $\begin{array}{c}\text { la, 'a ('a táula) } \\
\text { l' (l'acqua) }\end{array}$ & के \\
\hline masc. & $\overline{\mathbf{I}}_{\mathbf{L L}} \mathbf{\mathbf { O }} \mathbf{s}>$ & $\operatorname{los}$ & i, gli & li, 'i, l' ('i cani) & $\dot{g}$ \\
\hline fem. & $\check{I}_{\text {LLĀAs }}>$ & las & le & li, 'i, l' ('i táuli) & $\overline{2}$ \\
\hline
\end{tabular}

En siciliano, existen distinciones regionales de los artículos; en la isla se usan: lu, la y li. Algunas veces cae la <l> inicial. También se pierde la distinción de género en el plural. 
En italiano, se usa lo y gli antes de los sustantivos masculinos que empiezan con $<\mathrm{z}>$ o con $<\mathrm{s}+$ consonante $>$; por ejemplo se habla de lo zio (el tío) a quién le gusta lo sport (los deportes) y gli stranieri (los extranjeros). L' se usa ante los sustantivos que empiezan con vocal; por ejemplo, se habla de l'amiche (las amigas) que estudian en l'universitá (la universidad).

Los pronombres personales de primera y segunda persona singular del español, siciliano e italiano, provienen de los pronombres personales existentes en latín, y no presentan mucha dificultad en cuanto a su evolución etimológica. Yo, io e iu provienen del pronombre personal latino EGO; y tú, tu y tu provienen de TUU.

\begin{tabular}{|c|c|c|c|}
\hline Latín & Español & Italiano & Siciliano \\
\hline EGO & yo & io & iu \\
\hline TU & tú & tu & tu \\
\hline
\end{tabular}

En siciliano, los pronombres personales de primera y segunda persona plural son parecidos a los del español, por lo que provienen de la unión de los pronombres latinos nos y vos y el enfático ALTRUm latino. En español existen nos(otros) y vos(otros) y en siciliano tenemos nui(autri) y vui(autri). En cambio, en italiano, estos pronombres evolucionaron a noi y voi. En algunas regiones de Sicilia, también se usan las formas abreviadas nui y vui.

Los pronombres personales sujeto

\begin{tabular}{|c|c|c|}
\hline Español & Italiano & Siciliano \\
\hline yo & io & $\mathrm{iu}$ \\
\hline tú & $\mathrm{tu}$ & tu \\
\hline él & lui, (egli, esso) & iddu, lo, illu \\
\hline ella & lei, (ella, essa) & idda, la, illa \\
\hline nosotros & noi & nui, nuatri \\
\hline vosotros & voi & vui, vuatri \\
\hline ellos, ellas & loro, (essi, ese) & iddi, illi, li \\
\hline
\end{tabular}

Como se observa en el recuadro anterior, en la tercera persona, los pronombres provienen del ille latino, menos las formas italianas esso, essa, essi y ese, que se derivan del identificador enfático latino IPSE y se usan más que nada en el lenguaje formal. Se refieren generalmente a un sujeto no humano. De igual manera, los pronombres italianos egli y ella se restringen generalmente al uso literario, de manera que lui, lei y loro son las formas dominantes de uso común o regular en el lenguaje coloquial. En siciliano las formas más usadas son iddu, idda e iddi. 


\subsection{Diferencias morfológicas de número: los plurales}

En comparación con el italiano y el español, el siciliano presenta diferentes variaciones morfológicas o alomorfos para formar el plural. Tanto los plurales masculinos como los femeninos pueden terminar en $<i>$.

Estas terminaciones en $<\mathrm{i}>$ para el masculino y el femenino pueden crear confusión hasta para los mismos sicilianos; por ejemplo, tengo dos hijos y tres hijas se escribiría en siciliano aiu du' figghi e tri figghi. Para evitar la ambigüedad y facilitar la comunicación, se especifica el género añadiendo la aclaración de macho o hembra: aiu du’ figghi masculi e tri figghi fimmini. Como generalización de este fenómeno, se escucha también un figghiu masculu y na figghia fimmina que se tratan por tanto de sobrecorrecciones.

A continuación, se ofrece un resumen de las variantes más generales de los sustantivos plurales en siciliano; téngase en cuenta que se dan otras irregulares que no se muestran.

Los plurales de los sustantivos en siciliano

\begin{tabular}{|c|c|c|}
\hline & singular & plural \\
\hline masc. terminado en & $-\mathrm{u},-\mathrm{a},-\mathrm{i}$ & $-\mathrm{i}$ \\
\hline fem. terminado en & $\begin{array}{c}-\mathrm{a},-\mathrm{i} \\
-\mathrm{u}\end{array}$ & $\begin{array}{l}-\mathrm{i} \\
-\mathrm{u}\end{array}$ \\
\hline $\begin{array}{l}\text { masc. o fem. } \\
\text { terminado en }\end{array}$ & $\begin{array}{c}\text {-nca, -ncu } \\
\text {-nga, -ngu } \\
\text {-rca, -rcu } \\
\text {-rga, -rgu } \\
\text {-ca, -cu, -ga, -gu }\end{array}$ & $\begin{array}{c}\text {-nchi } \\
\text {-gni } \\
\text {-rchi } \\
\text {-rghi } \\
\text {-chi, -ghi }\end{array}$ \\
\hline
\end{tabular}

\subsection{Diferencias morfológicas: muy, molto, veru y el superlativo}

La palabra latina multum dio "mucho" y "muy" en español y el adjetivo molto en italiano, mientras que en siciliano no se mantuvo. En italiano, el adjetivo molto se usa como el adverbio "muy" del español, de modo que una ragazza molto dolce se traduce como "una chica muy dulce" en español, y na carusa veru duci en siciliano, donde veru equivale a muy y molto. En italiano también se utiliza el adjetivo vero, pero con un valor enfático, no tanto cuantificador o comparativo como molto.

Alternativamente, en siciliano se repite el adjetivo para intensificar lo expresado, es decir, se dobla el adjetivo para dar énfasis: "una chica muy dulce" se dice na carusa duci duci y "un chico muy grande" se dice 'n carusu `ranni ‘ranni.

168


El sufijo -issimo en italiano e "-ísimo" en español se usan semánticamente de la misma forma, de modo que "dulcísimo" y dolcissimo equivalen a "muy dulce", con un significado cuantificador e intensificador. Estos sufijos adjetivales vienen del superlativo latino -ISsimu que, al evolucionar, perdió su valor semántico de superlativo, aunque sí conservó su forma etimológica. Paralelamente, el valor del superlativo latino se sustituyó en español por la estructura sintáctica de un adverbio cuantificador, seguido del adjetivo con la preposición "de", por ejemplo: el más alto de, el menos inteligente de, etc. Es decir que la estructura sintética-morfológica latina del superlativo pasó a tener un patrón analítico-sintáctico.

El sufijo -issimu pocas veces se utiliza en siciliano. En cambio se emplea con frecuencia el prefijo stra- (que vendría a ser el "extra" del español) y los sufijos -idduni o -uni como se observa en los siguientes ejemplos.

Intensificadores de adjetivos

\begin{tabular}{|c|c|c|}
\hline Español & Italiano & Siciliano \\
\hline muy grande, grandísimo & molto grande, grandissimo & stragranni, straranni \\
\hline muy dulce, dulcísimo & molto dolce, dolcissimo & straduci [stradútfi] \\
\hline muy rico, riquísimo & molto riccho, ricchisimo & strarriccu \\
\hline muy viejo, viejísimo & molto vecchio, vecchisimo & $\underline{\text { straviechiu [stravjékju] }}$ \\
\hline muy feo, feísimo & molto brutto, bruttisimo & lariuni, lariulidduni \\
\hline
\end{tabular}

\subsection{Diferencias morfológicas: el futuro}

Se puede afirmar que el uso del futuro en siciliano es poco frecuente. Este fenómeno no resulta nuevo y, según Camilleri, se debe a varias razones: en primer lugar, postula que ocurre por el rechazo a la desinencia con vocales acentuadas (ya que suenan demasiado fuertes en siciliano); y en segundo lugar por la <r> que parece dificultar la pronunciación.

Esta tendencia es muy parecida a lo que ocurre en español, sobre todo en Latinoamérica, donde el futuro se sustituye con más y más frecuencia por el presente, ya sea simple o perifrástico ("voy a estudiar" por ejemplo). En español se usa la construcción ir $+a+$ infinitivo, como en "Luis va a llegar mañana" o con el verbo conjugado en presente con adverbios que indican futuro, como "llego mañana".

También en siciliano se utilizan estas dos formas, tanto el presente perifrástico, ir $+a+$ infinitivo, como el uso del presente simple; por ejemplo, en vaiu a nesciri (voy a salir) o en iddi arrivannu stasira (ellos llegan esta tarde). 
En italiano, el uso del futuro simple también está disminuyendo pero se da con frecuencia, lo que no ocurre en siciliano. Esto se debe seguramente al hecho de que no existe la construcción ir $+a+$ infinitivo en italiano. El futuro simple, de uso común tanto en italiano como en español, no se emplea, y se cree que nunca se ha empleado de ninguna forma en siciliano.

\begin{tabular}{|c|c|c|c|}
\hline & Español & Italiano & Siciliano \\
\hline normativo & Te escribiré apenas llegue & Ti scriverò appena sarò arrivato & $\begin{array}{c}\text { Ti scrivu } \\
\text { sppena arrivu }\end{array}$ \\
\hline coloquial & Te escribo apenas llegue & Ti scriverò appena arriverò & appena \\
\hline
\end{tabular}

\subsection{Diferencias sintácticas: la $a$ del objeto directo de persona}

El siciliano ha heredado del español el uso de la a acusativa, de objeto directo de persona, lo que no ocurre en italiano. En español "María llama a su hijo" se dice en siciliano María chiama a so figghiu mientras que en italiano es María chiama suo figlio. De igual manera, en español se dice "María ama a su marido" y en siciliano María ama a so maritu, pero en italiano María ama suo marito.

No obstante, esta diferencia sintáctica entre el siciliano y el italiano se está perdiendo. La similitud sintáctica entre el español y el siciliano en estructuras como éstas es cada vez menor, dada la influencia tan aplastante del italiano en el siciliano moderno.

A diferencia del español, en siciliano, la a acusativa no se usa (ni nunca se usó), cuando se habla de animales o de conceptos u objetos personificados, de manera que "María quiere a su perro" en siciliano es María ama u so cani; o "Fabio ama a su mar" es Fabio ama u so mari.

\section{Conclusión}

Además de destacar algunas de las similitudes que existen entre el español y el siciliano, esta investigación se ha centrado en resaltar las diferencias gráficas, fonéticas y morfológicas de las tres variantes lingüísticas originarias del latín: español, italiano y siciliano. Las raíces comunes en el latín vulgar, más la influencia árabe, resultan quizás las características que más correspondencias han producido. Los cognados del latín, del árabe y del griego facilitan la posibilidad de comunicación entre sicilianos e hispanohablantes, aun sin haber adquirido la lengua. Este cotejo de voces pretende presentar, de una forma clara y

-


sucinta, comparaciones importantes entre las variedades estudiadas, sin ánimo de profundizar en la diacronía o en la historia evolutiva de cada vocablo per se.

A pesar de que el siciliano está considerado por muchos expertos un dialecto del italiano, se ha observado que mientras que el desarrollo del siciliano a veces refleja los mismos cambios fonéticos y morfológicos del italiano (o del español), otras sufrió cambios únicos y muy marcados, con un desarrollo independiente de otras variantes romances. Como se ha demostrado con varios ejemplos, el siciliano resulta a veces la variante que más similitud ha guardado con el latín; esto se ha ilustrado con los pronombres personales que provienen de ILLUM, (la <-u> final se mantiene); con algunas palabras latinas que perduraron en siciliano, pero que se perdieron o se reemplazaron en otras lenguas romances (como requia, paz); con los numerosos cambios fonéticos, semánticos y morfológicos que lo conectan más estrechamente a su raíz latina.

Esta transformación peculiar, dentro del marco romance europeo, ha provocado que algunos académicos defiendan al siciliano como lengua independiente, y no como dialecto del italiano. Ya se clasifique de lengua, ya de dialecto, el siciliano se presenta como una fuente de gran valor lingüístico y, dadas las pocas investigaciones descriptivascomparativas entre el español y el siciliano, su contraste nos permite ofrecer una perspectiva pionera. Su estatus político como dialecto del italiano y su poca aceptación entre los jóvenes (quienes prefieren hablar la lengua oficial), pueden llevar a la desaparición de esta variante, por lo que desde aquí proponemos fomentar más estudios y realzar la tras-

cendencia lingüística del siciliano, en el campo de las investigaciones románicas.

\section{Bibliografía}

Bellestri, Joseph (1985). Basic Sicilian-English Dictionary. Ann Arbor: Joseph Bellestri.

Bonfante, Giuliano (1986). La continuità del latino in Sicilia. Mediterranean Language Review, 2, 46-49.

Camilleri, Salvatore (2002). Grammatica siciliana. Catania, Italia: Boemi.

Cano Aguilar, Rafael (1999). El español a través de los tiempos. Madrid: Arco Libros. 
Estudio lingüístico-comparativo del siciliano y el español / Eva Núñez, Raven Chakerian

Chiuchiu, Angelo (2007). Grammatica italiana per stranieri. Perugia, Italia: Edizione Guerra.

Colón y Domènech, Germà (1976). Léxico catalán en la Romania. Madrid: Gredos.

(1997). Estudis de filologia catalana i románica. Barcelona: Institut interuniversitari de Filologia Valenciana i de Publicaciones de l'Abadia de Montserrat.

Corriente, Federico (1999). Diccionario de arabismos y voces afines en iberorromance. Madrid: Gredos.

Devoto, Giacomo (1978). The Languages of Italy. (Trad. V. Louise Katainen). Chicago: University of Chicago Press.

Elcock, William D. (1960). The Romance Languages. London: Faber.

Fortuna, Antonella (2002). Grammatica siciliana: principali regole grammaticali, fonetiche e grafiche (comparate tra i vari dialetti siciliani). Caltanissetta, Italia: Terzo Millennio.

García Sánchez, Jairo (2007). Atlas toponímico de España. Madrid: Arco Libros.

Guarneri, Marco (1997). Hippocrene Concise Dictionary: Sicilian-English, English-Sicilian. New York: Hippocrene.

Guerriero, Alfonso (1998). Arabic Place-Names: A Historic Journey through Sicily. The American Society of Geolinguistic, 24, 7785.

Hualde, José Ignacio, Antxón Olarrea y Anna María Escobar (2001). Introducción a la lingüística hispánica. Cambridge: Cambridge University Press.

Lapesa, Rafael (1995). Historia de la lengua española. Madrid: Gredos.

Lüdtke, Helmut (1968). El beréber y la lingüística románica. XI Congreso internacional de lingüística y filología románicas, II. Madrid, CSIC, 2, 467-72.

Maiden, Martin (1995). A Linguistic History of Italy. London: Longman Group Limited.

Mazzola, Michael Lee (1976). Proto-Romance and Sicilian. Lisse, Bélgica: Peter Rider Press.

Noll, Volker (2006). La aglutinación del artículo árabe al en el léxico español. Cosmos Léxico, XIV, 35-49. 
Núñez Méndez, Eva (2012). Fundamentos teóricos y prácticos de historia de la lengua española. New Haven: Yale University Press.

Privitera, Joseph F. (2004). Sicilian: The Oldest Romance Language. Ottawa, Canadá: Legas Press.

Resnick, Melvyn C. (1981). Introducción a la historia de la lengua española. Washington D. C.: Georgetown University Press.

Rohlfs, Gerhard (1966). Grammatica Storica della Lingua Italiana e dei suoi Dialletti. (Trad. Salvatore Persichino). Torino, Italia: Giulio Einaudi Editore.

Sucato, Ignazio (1975). La lingua siciliana: origine e storia (2a Ed.). Palermo, Italia: Scuola Grafica Salesiana.

Tornatore, Mathew G. C. (2003). El siciliano y su herencia lingüística española. Nueva York: Edwin Mellen Press. 